\title{
A statistical mechanics view on Kitaev's proposal for quantum memories
}

\author{
R. Alicki ${ }^{\dagger}$, M. Fannes ${ }^{\ddagger}$ and M. Horodecki ${ }^{\dagger}$ \\ $\dagger$ Institute of Theoretical Physics and Astrophysics \\ University of Gdańsk, Poland \\ $\ddagger$ Instituut voor Theoretische Fysica \\ K.U.Leuven, Belgium
}

\begin{abstract}
We compute rigorously the ground and equilibrium states for Kitaev's model in 2D, both the finite and infinite version, using an analogy with the 1D Ising ferromagnet. Next, we investigate the structure of the reduced dynamics in the presence of thermal baths in the Markovian regime. Special attention is paid to the dynamics of the topological freedoms which have been proposed for storing quantum information.
\end{abstract}

\section{Introduction}

Despite the enormous activity in the field of quantum information in the last decade, the fundamental problem of how to protect quantum information for macroscopic periods of time remains open. In contrast to the popular belief the Fault Tolerant Quantum Computation (FTQC) ideas [1, 2, 3, 4, 5] do not provide the final solution to this problem. The mathematical models behind these schemes are highly oversimplified, phenomenological, and do not take into account the restrictions imposed by the laws of thermodynamics. As FTQC involves time-dependent control by external fields the rigorous analysis of the corresponding models which can be derived from first principles is extremely difficult. In particular, the standard approximations based on the rigorous versions of Markovian limits (weak coupling or low density $[6,7,8]$ ), which yield descriptions consistent with thermodynamics, cannot be applied, see $[9,10,11,12,13]$ in this context. Therefore, the approach to quantum memories based on the idea of self-correcting systems is more promising for rigorous studies. It deals with $N$-qubit systems with specially designed Hamiltonians which are supposed to stabilise an algebra of observables corresponding to one or few qubits, at least below some critical 
temperature. The construction of self-correcting Hamiltonians is based on the properties of error correcting codes and the most advanced examples are 2, 3 and 4D Kitaev models [14, 15] associated with toric codes.

It is instructive to discuss first the mechanism of protection of classical information using phase transitions in quantum systems. In the general approach based on the algebraic formalism for infinite systems, i.e. systems in the thermodynamic limit, we characterise equilibrium states by the KMS condition [16]. States that satisfy the KMS condition are known to have good stability properties such as passivity [17]. Typically, one expects that for sufficiently high temperatures there exists a unique KMS state, while below a certain critical temperature $T_{c}$ a simplex of KMS states can appear. The extreme states which span this simplex correspond to pure thermodynamical phases labelled by the values of the order parameters. They can be used to encode classical information.

In a complementary dynamical picture we consider an $N$-particle system weakly interacting with a heat bath at temperature $T$. The finite system possesses a unique equilibrium state given by a Gibbs density matrix. However, for systems exhibiting phase transitions and for $T<T_{c}$ the unique equilibrium state can be decomposed into many long-living metastable states corresponding to local minima of the free energy. These minima are separated by free energy barriers of heights proportional to $N$. This makes transitions caused by thermal fluctuations highly improbable and leads to life-times exponentially increasing with $N$. Obviously, the metastable states should become equilibrium (KMS) states in the limit $N \rightarrow \infty$. Although the equivalence of equilibrium and dynamical pictures is generally accepted there exist only few rigorous results that support this, e.g. the mean-field Curie-Weiss model [18] and Bose-Einstein condensation [19].

From the discussion of above it follows that extending these ideas to the field of quantum information in order to construct quantum memories is not possible. The encoding of qubits into different thermodynamical phases of an infinite system is impossible because of the simplex structure of the KMS states. A simplex is a purely classical state space and cannot even support a single qubit.

Actually, in the literature one attempts to construct self-correcting Hamiltonians for $N$ qubits in such a way that their degenerated ground states correspond to codewords encoding states of one or few qubits $[14,15]$. How- 
ever, good codewords are locally indistinguishable, which implies that in the thermodynamic limit all of them merge into a single ground state of the infinite system. Therefore one doesn't expect that such systems exhibit phase transitions at any temperature and so cannot even support a classical memory. Such a view is consistent with Pirogov-Sinai [20] type results where one shows that well-separated ground states of infinite classical systems extend to separated phases at sufficiently low temperatures.

A possible objection to the previous reasoning, which was presented in [25], is that the mathematical framework of infinite systems, used to describe the thermodynamic limit, is too simplistic. One cannot exclude that observables which distinguish between the degenerated ground states of the finite system but disappear in the thermodynamic limit are long-lived in the presence of a generic heat bath at sufficiently low temperatures. This would imply that the expected equivalence of the infinite system equilibrium picture and the dynamical picture is not universal and that a new type of "purely dynamical phase transitions" exists. In order to prove or disprove this claim one should study finite supposedly self-correcting systems weakly coupled to generic models of heat baths and search for possibly slowly relaxing degrees of freedom. These could then support states of encoded qubits for times exponentially increasing with $N$.

It is interesting to compare the problem of stable quantum memories to another long-standing problem of quantum mechanics: the absence of certain superpositions of quantum states for sufficiently large systems. Well-known examples are Schrödinger cat states, the appearance of a classical world, the problem of molecular structure, chiral molecules, deformed nuclei, ... [21] It seems to be generally accepted that the main ingredient for the solution of this problem is the stability of quantum states of the observed system with respect to the coupling to an environment, sometimes identified with a measuring apparatus or even a conscious observer. As in the case of quantum memories two approaches have been developed, a static and a dynamical one. The first relies on dressing the states of the bare system. This can produce disjoint stable states of the dressed system when using environments with an infinite number of degrees of freedom and leads to unobservable superpositions, at least for coupling constant larger then a certain critical value. The mathematical mechanism is completely similar to that which is responsible for the appearance of disjoint KMS states in the thermodynamic limit. The dynamical approach, on the other hand, refers to decoherence phenomena 
which transform superpositions of states into mixtures with respect to a certain particularly stable pointer basis. The relations between both pictures and their ranges of applicability are still a topic of debate. Unfortunately, except for the spin-boson model [22] no rigorous result has been obtained.

The purpose of this paper is to start a systematic and rigorous comparison of the infinite system equilibrium picture and the finite system dynamical picture for models designed to support quantum memories. We restrict ourselves to the 1D (quantum) Ising model and the 2D Kitaev model. Although there exist heuristic arguments that, in spite of earlier claims concerning the $2 \mathrm{D}$ case $[14,23,24]$, both models cannot provide stable quantum memories [25] it is instructive to develop the basic mathematical tools for analysing these relatively simple cases, hoping that they will be useful for $3 \mathrm{D}$ or $4 \mathrm{D}$ models. Only the last case is presently considered by some experts to be a proper candidate for a quantum memory [15]. One should mention that formally the 1D quantum Ising model can be considered to be the 1D Kitaev model and hence the parallel analysis of both could be helpful for further studies.

The main simplifying feature of the Kitaev models is the absence of wave propagation. This leads to a discrete spectrum of the Hamiltonian with level spacings independent of the size of the system. This property allows to apply the Markovian approximation in terms of Davies weak coupling limit which reproduces fully the expected phenomenology of an open system weakly coupled to a heat bath. The Davies semigroup generators possess a simple local structure and behave well with respect to the thermodynamic limit. Moreover, for Kitaev's model we can determine the structure of the algebras which can encode qubits and we obtain the dynamics of the qubit observables.

\section{Ground and equilibrium states of 1D Ising and 2D Kitaev models}

The physical substrate which is to carry the qubits has basic spin observables that are attached to the sites of a chain, a ring, a lattice ... We will describe their basic observables by Pauli matrices $\sigma_{j}^{\alpha}$ where $j$ points at the site at which the spin lives and $\alpha=x, y, z$. These basic observables obey the 
relations

$$
\begin{aligned}
& \sigma_{j}^{x} \sigma_{j}^{y}=i \sigma_{j}^{z} \quad \text { and cyclic permutations of } x, y, z \\
& \left(\sigma_{j}^{\alpha}\right)^{\dagger}=\sigma_{j}^{\alpha} \quad \text { and } \quad\left(\sigma_{j}^{\alpha}\right)^{2}=\mathbb{1} \\
& {\left[\sigma_{j}^{\alpha}, \sigma_{k}^{\beta}\right]=0 \quad \text { for } j \neq k .}
\end{aligned}
$$

For systems with a finite number of spins the energy of the system is encoded in the Hamiltonian and physically relevant states are ground and thermal states. The first are determined by the eigenstates of the Hamiltonian corresponding to the lowest energy and the second are given by the Gibbs canonical density matrix at temperature $T$. The limit $T \rightarrow 0$ of the Gibbs state is a ground state. In degenerate cases this limit is the average of all ground states and there are therefore generally more ground states than just the low temperature limit of the canonical Gibbs state.

Typically one is interested in quantities that have a nice scaling behaviour with respect to $N$ and hence in the leading asymptotic behaviour. This is precisely what the formalism of systems with an infinite number of particles catches: it provides right away a framework that allows to define and compute the asymptotic ground and equilibrium states. Obviously the total energy of an infinite systems is not a sensible observable and one characterises ground and equilibrium states in terms of the interactions between the spins. In fact sufficiently local interactions generate a well-behaved dynamics on the infinite system given by a group of automorphisms $\left\{\alpha_{t} \mid t \in \mathbb{R}\right\}$ of the observables. The generator of this group is a derivation $\delta$ determined by $\alpha_{t}=\exp (i t \delta)$. It satisfies Leibniz's rule $\delta(X Y)=\delta(X) Y+X \delta(Y)$ and is self-adjoint $\delta\left(X^{\dagger}\right)=-(\delta(X))^{\dagger}$. For a finite system with Hamiltonian $H$ one has $\delta(\cdot)=[H, \cdot]$.

There are various ways of introducing ground and equilibrium states of an infinite system, such as thermodynamical limits of local Gibbs states possibly with boundary conditions or as states maximising the entropy density for a given internal energy density. We shall use the characterisation in terms of the dynamics. This leads to the ground state equation and the KMS condition. For finite systems these conditions return the usual notions of ground state and canonical Gibbs state at a given temperature.

A state $\omega$ satisfies the ground state equation if

$$
\omega\left(X^{\dagger} \delta(X)\right) \geq 0
$$


It satisfies the KMS condition at inverse temperature $\beta=1 / k_{\mathrm{B}} T>0$ if there exists for any choice of observables $X$ and $Y$ a function

$$
F_{X, Y}:\{z \in \mathbb{C} \mid 0 \leq \Im \mathfrak{m} \leq \beta\} \rightarrow \mathbb{C}
$$

such that $F_{X, Y}$ is analytic in the open strip $\{z \in \mathbb{C} \mid 0<\Im \mathfrak{m}<\beta\}$, is bounded in the closed strip, extends continuously to the lines $\Im \mathfrak{m} z=0$ and $\Im \mathfrak{m}=\beta$ and satisfies for $t \in \mathbb{R}$

$$
F_{X, Y}(t)=\omega\left(X \alpha_{t}(Y)\right) \quad \text { and } \quad F_{X, Y}(t+i \beta)=\omega\left(\alpha_{t}(Y) X\right) .
$$

Loosely speaking, the KMS condition says that

$$
\omega\left(X \alpha_{i \beta}(Y)\right)=\omega(Y X)
$$

at least if the expression $\alpha_{i \beta}(Y)$ makes sense. It therefore links equilibrium states with the Heisenberg dynamics at imaginary times. For finite systems when the dynamics is given by

$$
\alpha_{t}(X)=\mathrm{e}^{i t H} X \mathrm{e}^{-i t H}
$$

it follows readily from (7) that only the canonical Gibbs state at inverse temperature $\beta$ satisfies the KMS condition.

If the system has a local symmetry in the sense that there exists a local unitary $U$, i.e. a unitary living on a finite number of sites, such that

$$
\alpha_{t}\left(U X U^{\dagger}\right)=U \alpha_{t}(X) U^{\dagger}
$$

and if $\omega$ is a KMS state, then also the transformed state $\mathrm{X} \mapsto \omega\left(U X U^{\dagger}\right)$ is a KMS state at the same inverse temperature $\beta$ and is unitarily equivalent to the original one. A general property of KMS states, see[16], implies that $\omega$ is actually invariant under the symmetry

$$
\omega(X)=\omega\left(U X U^{\dagger}\right) .
$$

There is no such result for ground states as determined by equation (4).

We shall consider two models: the Ising ferromagnet and a model introduced by Kitaev. Both models have a finite version on a ring and a torus respectively. We shall also consider the infinite versions, they live on a chain and on a staggered square lattice. The particle interactions are in both cases nearest-neighbour and both models, either finite or infinite, allow for ground states in a very strong sense in that the states minimise all local interactions. This can be seen as a complete absence of frustration. 


\subsection{D Ising ferromagnet}

The interaction for an Ising ferromagnet is given in terms of bonds

$$
Z_{b}:=\sigma_{j}^{z} \sigma_{j+1}^{z}, \quad \text { with } b=\{j, j+1\}
$$

In this expression $j$ is either a site of a ring or a chain.

Let us first consider the finite system of $N$ spins on a ring, meaning that we identify the sites 1 and $N+1$. Clearly the bond spins are not independent as they satisfy the relation

$$
\prod_{b} Z_{b}=\mathbb{1}
$$

Any $(N-1)$ independent bond observables - one can omit an arbitrary one due to (12) - correspond to the $\sigma^{z}$ observables of $(N-1)$ qubits. The algebra $\mathcal{A}_{\mathrm{z}}$ generated by the bonds is Abelian but is not generated by a maximal set of commuting observables in the algebra of the $N$ spins. In fact its commutant $\mathcal{A}_{\mathrm{z}}^{\prime}$ within the spin algebra is the product of a qubit algebra and $\mathcal{A}_{\mathrm{z}}$. This can be seen by the following direct construction. Choose

$$
\mathbf{X}=\sigma_{1}^{x} \sigma_{2}^{x} \cdots \sigma_{N}^{x}, \quad \mathbf{Y}=\sigma_{1}^{y} \sigma_{2}^{x} \cdots \sigma_{N}^{x}, \quad \text { and } \mathbf{Z}=\sigma_{1}^{z} .
$$

The matrices $\mathrm{X}, \mathrm{Y}$ and $\mathrm{Z}$ satisfy the same relations as the Pauli matrices and commute with the algebra of the bond observables. It can be checked that any element commuting with $\mathcal{A}_{\mathrm{z}}$ belongs to the algebra generated by the observables of the encoded qubit (13) and $\mathcal{A}_{\mathrm{z}}$.

Let us now turn to the infinite system which lives on a chain. The local observables are finite linear combinations of finite products of single site spin observables and the quasi-local observables are obtained by completing this local algebra with respect to the natural norm. The completion of the algebra of the bond observables $\mathcal{A}_{\mathrm{z}}$ is again commutative but this time the commutant within the quasi-local algebra is also Abelian, it is generated by e.g. $\sigma_{1}^{z}$ and $\mathcal{A}_{\mathrm{z}}$. The reason for this simplification is to be found in the disappearance of the infinite product of $\sigma^{x}$ observables in the limit $N \rightarrow \infty$ for the $\mathrm{X}$ and $\mathrm{Y}$ observables in (13). From a mathematical point of view such a limit does not exist in the spin algebra, from a physical point of view the distribution of such observables has no stable limit in any physically reasonable state. 
We shall examine the ground and equilibrium states of the Ising Hamiltonian with local Hamiltonian

$$
H_{\Lambda}^{\mathrm{Ising}}=-\sum_{b \subset \Lambda} Z_{b}
$$

Here $\Lambda$ is any finite subset of the chain.

\subsubsection{Ground states}

To compute the ground states we can use the following property of a state: suppose that $X=X^{\dagger}$ such that $X \leq \mathbb{1}$ and that $\omega(X)=1$, then $\omega(Y X)=$ $\omega(X Y)=\omega(Y)$ for any $Y$. Indeed, $0 \leq \mathbb{1}-X$ and so we can extract the square root of $\mathbb{1}-X$ and apply the Cauchy-Schwartz inequality

$$
|\omega(Y(\mathbb{1}-X))|^{2} \leq \omega\left(Y(\mathbb{1}-X) Y^{\dagger}\right) \omega(\mathbb{1}-X)=0 .
$$

Similarly $\omega(X Y)=\omega(Y)$.

Suppose now that $\omega$ is a state on the Abelian algebra $\mathcal{A}_{\mathrm{z}}$ generated by the bond observables $Z_{b}$ such that $\omega\left(Z_{b}\right)=1$ and let $\tilde{\omega}$ be an extension of this state to the spin algebra. As $Z_{b} \leq \mathbb{1}$, we can apply the argument of above and conclude that for any element $X$ in the spin algebra and any bond $b \tilde{\omega}\left(X Z_{b}\right)=\tilde{\omega}\left(Z_{b} X\right)=\tilde{\omega}(X)$.

This implies that $\tilde{\omega}$ is a ground state in the sense of (4). It suffices to show that $\tilde{\omega}\left(X^{*} \delta(X)\right) \geq 0$ for any local observable $X$. For such observables the action of the derivation $\delta$ is given by a finite sum of commutators

$$
\delta(X)=-\sum_{b \in \Lambda}\left[Z_{b}, X\right]
$$

Here $\Lambda$ is the set of bonds which have a non-empty intersection with the dependency set of $X$. We now compute

$$
\begin{aligned}
\tilde{\omega}\left(X^{\dagger} \delta(X)\right) & =\sum_{b \in \Lambda} \tilde{\omega}\left(X^{\dagger} X Z_{b}-X^{\dagger} Z_{b} X\right) \\
& =\sum_{b \in \Lambda}\left(\tilde{\omega}\left(X^{\dagger} X\right)-\tilde{\omega}\left(X^{\dagger} Z_{b} X\right)\right) \\
& =\sum_{b \in \Lambda} \tilde{\omega}\left(X^{\dagger}\left(\mathbb{1}-Z_{b}\right) X\right) \geq 0 .
\end{aligned}
$$


To compute the ground state expectation of an arbitrary spin observable for the finite system we consider arbitrary monomials in Pauli matrices. Using

$$
\tilde{\omega}\left(\sigma_{j}^{x}\right)=-\tilde{\omega}\left(Z_{b} \sigma_{j}^{x} Z_{b}\right)=-\tilde{\omega}\left(\sigma_{j}^{x}\right)
$$

for a site $j$ which belongs to the bond $b$ and the obvious extensions to arbitrary polynomials, we conclude that only monomials belonging to the commutant of $\mathcal{A}_{\mathrm{z}}$ can have non-zero expectation values. Moreover with $\mathrm{X}$ as in (13)

$$
\tilde{\omega}(\mathbf{X} B)=\tilde{\omega}(\mathbf{X}) \omega_{0}(B), \quad B \in \mathcal{A}_{z},
$$

and similar expressions replacing $\mathrm{X}$ by $\mathrm{Y}$ or $\mathrm{Z}$. The state $\omega_{0}$ is the unique state on $\mathcal{A}_{\mathrm{z}}$ that assigns the value 1 to all products of bond observables. We have therefore determined all ground states of the finite model: they are products of an arbitrary state on the qubit algebra and the state $\omega_{0}$.

The infinite system can be handled in exactly the same way, we now obtain product states of a classical bit with the state $\omega_{0}$. In particular, this implies that the set of ground states of the Ising ferromagnet on an infinite chain is a simplex, consisting only of mixtures of two extremal states. In principle, this could be used to encode a classical bit. In fact, this is a bit too optimistic as it is well-known for this model that at finite temperatures there is a unique equilibrium state.

\subsubsection{Equilibrium states}

Determining the equilibrium states of the finite and infinite Ising models as solutions of the KMS condition can be carried out along the same lines as in the previous section. Using for any site $j$ belonging to a bond $b$

$$
Z_{b} \sigma_{j}^{x} Z_{b}=-\sigma_{j}^{x} \quad \text { and } \quad Z_{b} \sigma_{j}^{y} Z_{b}=-\sigma_{j}^{y}
$$

and the general result about KMS states mentioned in (10) we conclude that the only monomials that have non-zero expectation values belong to the commutant of $\mathcal{A}_{\mathrm{z}}$. The solution of the KMS condition is the extension of the equilibrium state for the bond observables to $\mathcal{A}_{\mathrm{z}}^{\prime}$ assigning zero values to any observable of the form $\mathrm{X} B, \mathrm{Y} B$ or $\mathrm{Z} B, B \in \mathcal{A}_{\mathrm{z}}$ for the finite system and zero value to $\sigma_{1}^{z} B$ for the infinite system. 


\subsection{Kitaev's model in 2D}

The finite version of Kitaev's model lives on a $K \times K$ lattice with periodic boundary conditions (toroidal lattice), see the square dots in Fig. 1. The spins live on the edges of the lattice as shown by the black and white dots in the figure and so we have $N=2 K^{2}$ qubits. The interactions between the spins are given by star and plaquette terms $X_{s}$ and $Z_{p}$. A star is a diamond whose vertical vertices lie on the lattice of black dots, while the vertical vertices of a plaquette lie on the lattice of white dots. The actual observables are then

$$
X_{s}=\prod_{j \in s} \sigma_{j}^{x} \quad \text { and } \quad Z_{p}=\prod_{j \in p} \sigma_{j}^{z}
$$

The names star and plaquette correspond to the squares and stars marked by thick lines in the figure.

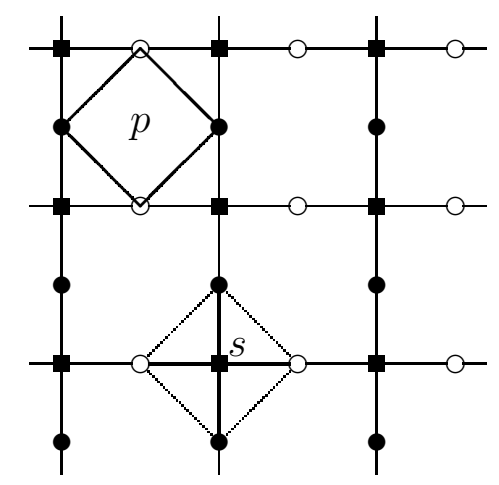

Fig 1: Kitaev's lattice

As stars and plaquettes have either 0 or 2 sites in common, $\left[X_{s}, Z_{p}\right]=0$. So, the algebras $\mathcal{A}_{\mathrm{x}}$ and $\mathcal{A}_{\mathrm{z}}$ generated by the $X_{s}$ and $Z_{p}$ are Abelian. We shall denote by $\mathcal{A}_{\mathrm{xz}}$ the algebra generated by $\mathcal{A}_{\mathrm{x}}$ and $\mathcal{A}_{\mathrm{z}}$. Because of the periodic boundary conditions the star and plaquette observables are not independent, they satisfy the relations

$$
\prod_{s} X_{s}=\mathbb{1} \quad \text { and } \quad \prod_{p} Z_{p}=\mathbb{1}
$$


otherwise they are independent. As in the Ising case the algebra $\mathcal{A}_{\mathrm{xz}}$ is not maximally Abelian within the spin algebra. Its commutant consists of a product of two qubit algebras and $\mathcal{A}_{\mathrm{xz}}$. This can again be seen quite explicitly by the following direct construction. Similarly to (13), one can introduce observables for two encoded qubits

$$
\begin{array}{ll}
\mathrm{Z}_{1}=\prod_{j \in c_{1}} \sigma_{j}^{z}, & \mathrm{Z}_{2}=\prod_{j \in c_{2}} \sigma_{j}^{z} \\
\mathrm{X}_{1}=\prod_{j \in c_{1}^{\prime}} \sigma_{j^{\prime}}^{x}, & \mathrm{X}_{2}=\prod_{j \in c_{2}^{\prime}} \sigma_{j^{\prime}}^{x}
\end{array}
$$

Here $c_{1}, c_{1}^{\prime}, c_{2}$ and $c_{2}^{\prime}$ are the loops shown in Fig. 2.

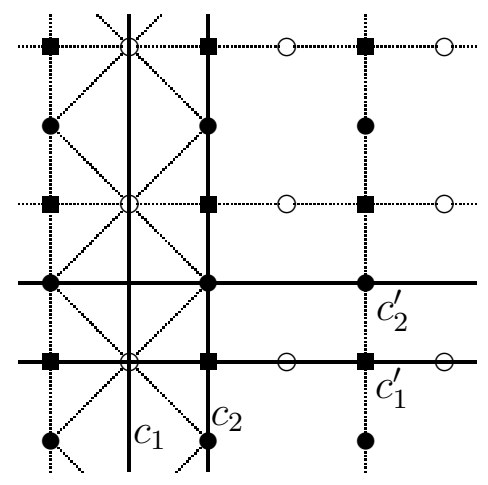

Fig 2: The loops in the toric lattice

Let us now turn to the infinite system which lives on an infinite staggered lattice. In contrast to the Ising case the commutant of the algebra $\mathcal{A}_{\mathrm{xz}}$ in the spin algebra is now $\mathcal{A}_{\mathrm{xz}}$ itself. This can again be understood in terms of the non-existence of the limits of the loop operators in (25) when $N \rightarrow \infty$. Indeed with increasing $N$, the length $K$ of the loops increases as $N^{1 / 2}$.

The local Hamiltonians of the model are

$$
H_{\Lambda}^{\mathrm{Kitaev}}=-\sum_{\{s \mid s \subset \Lambda\}} X_{s}-\sum_{\{p \mid p \subset \Lambda\}} Z_{p}
$$

Here $\Lambda$ is any finite subset of the staggered lattice. 


\subsubsection{Ground states}

Let $\omega_{0}$ be the state on the Abelian algebra $\mathcal{A}_{\mathrm{xz}}$ generated by the $\mathcal{A}_{\mathrm{x}}$ and $\mathcal{A}_{\mathrm{z}}$ such that

$$
\omega_{0}\left(X_{s}\right)=\omega_{0}\left(Z_{p}\right)=1 \text {. }
$$

Using the same arguments as for the Ising model we obtain that $\omega_{0}$ extends to a product state of a two qubit algebra and $\mathcal{A}_{\mathrm{xz}}$ for the finite model. For the infinite model there is only a unique extension $\tilde{\omega}$ as we argue in the following lines and this has, by unicity, to be pure. As before, we compute the expectation values of a finite product of elementary spin observables using (15). Consider a product of Pauli matrices living at the sites of a finite set $\Lambda$. Consider first the point of $\Lambda$ farthest to the North and also, on the row to which it belongs, farthest to the East. Suppose that this point belongs to the lattice of black dots. An argument similar to that in (20) kills the expectation of the observable unless it carries a $\sigma^{z}$ Pauli matrix. Suppose it does and consider then the point on the lattice of dots, lying South-East of the first. Unless this carries also a $\sigma^{z}$ spin the expectation also vanishes because of anticommutation with a suitably chosen plaquette observable. If both points carry such $\sigma^{z}$ matrices, then they can be removed by multiplying with a plaquette observable having the two points as North-East boundary. A similar argument holds if our original point belonged to the lattice of white dots. Continuing like this we see that the only observables having non-zero expectation value are products of star and plaquette observables, but all these have expectation 1 because of (15). So this model has a unique ground state and cannot be used to encode even a single classical bit.

It remains to show that indeed a state satisfying (27) exists. It suffices therefore to observe that we can map the star observables on spins of an Ising model on $\mathbb{Z}^{2}$ and the plaquettes on these of a similar Ising model. Doing so, the interaction in the Kitaev model just becomes that of a couple of free Ising spin systems on $\mathbb{Z}^{2}$, which clearly admits such a ground state.

\subsubsection{Equilibrium states}

Probably the most important feature of the plaquette and star observables is that

$$
\left[X_{s}, X_{s^{\prime}}\right]=\left[X_{s}, Z_{p}\right]=\left[Z_{p}, Z_{p^{\prime}}\right]=0
$$


for all choices of $s, s^{\prime}, p$ and $p^{\prime}$. Moreover,

$$
X_{s}=X_{s}^{\dagger}, \quad Z_{p}=Z_{p}^{\dagger}, \quad \text { and } X_{s}^{2}=Z_{p}^{2}=\mathbb{1}
$$

Therefore, for the infinite system, the algebra generated by the $X_{s}$ and $Z_{p}$ is isomorphic to the algebra of continuous function on a configuration space which is a countable product of copies of Ising spaces $\{\uparrow, \downarrow\}$. We can, moreover, choose the isomorphism in such a way that each of the $X_{s}$ and $Z_{p}$ is mapped onto an Ising variable.

Let $\Lambda \subset \mathbb{Z}^{2}$ be a finite set. Adding successively boundary layers, we obtain a collection of sets

$$
\begin{aligned}
& \Lambda(0):=\Lambda \\
& \Lambda(1):=\left(\bigcup_{s \cap \Lambda(0) \neq \emptyset} s\right) \bigcup\left(\bigcup_{p \cap \Lambda(0) \neq \emptyset} p\right) \\
& \Lambda(2):=\left(\bigcup_{s \cap \Lambda(1) \neq \emptyset} s\right) \bigcup\left(\bigcup_{p \cap \Lambda(1) \neq \emptyset} p\right)
\end{aligned}
$$

In the Heisenberg picture, a local observable $X$ initially living in a finite set $\Lambda$ evolves after time $t$ into

$$
\alpha_{t}(X):=X+i t \delta(X)+\frac{(i t)^{2}}{2 !} \delta^{2}(X)+\cdots
$$

where the derivation $\delta$ is obtained from the local Hamiltonians

$$
\begin{aligned}
\delta(X) & =\lim _{M \rightarrow \mathbb{Z}^{2}}\left[H_{M}^{\mathrm{Kitaev}}, X\right] \\
& =-\sum_{s \subset \Lambda(1)}\left[X_{s}, X\right]-\sum_{P \subset \Lambda(1)}\left[Z_{p}, X\right] \\
& =\left[H_{\Lambda(1)}^{\mathrm{Kitaev}}, X\right] .
\end{aligned}
$$


Using the commutation relations between the $X_{s}$ and $Z_{p}$, we see that

$$
\begin{aligned}
\delta^{2}(X) & =-\sum_{s \subset \Lambda(2)}\left[X_{s}, \delta(X)\right]-\sum_{p \subset \Lambda(2)}\left[Z_{p}, \delta(X)\right] \\
& =-\sum_{s \subset \Lambda(2)} \sum_{s^{\prime} \subset \Lambda(1)}\left[X_{s},\left[X_{s^{\prime}}, X\right]\right]-\cdots \\
& =-\sum_{s^{\prime} \subset \Lambda(1)} \sum_{s \subset \Lambda(2)}\left[X_{s^{\prime}},\left[X_{s}, X\right]\right]-\cdots \\
& =-\sum_{s^{\prime} \subset \Lambda(1)} \sum_{s \subset \Lambda(1)}\left[X_{s^{\prime}},\left[X_{s}, X\right]\right]-\cdots
\end{aligned}
$$

We therefore have for any $k=1,2, \ldots$

$$
\delta^{k}(X)=\left[H_{\Lambda(1)}^{\text {Kitaev }},\left[H_{\Lambda(1)}^{\text {Kitaev }}, \cdots\left[H_{\Lambda(1)}^{\text {Kitaev }}, X\right] \cdots\right]\right] \quad k \text { commutators. }
$$

Hence, for all $t \in \mathbb{R}, \alpha_{t}(X)$ lives in $\Lambda(1)$.

The commutation of the $X_{s}$ and $Z_{p}$ also allows us to conclude that for any $s$ and $p$ and any quasi-local observable $X$

$$
\alpha_{t}\left(\left[X_{s}, X\right]\right)=\left[X_{s}, \alpha_{t}(X)\right] \quad \text { and } \quad \alpha_{t}\left(\left[Z_{p}, X\right]\right)=\left[Z_{p}, \alpha_{t}(X)\right] .
$$

Because of (42), given an $\alpha_{t}$-KMS state $\omega$, for any choice of $s$ and $p$ and for any $s \in \mathbb{R}$, also

$$
X \mapsto \omega\left(\mathrm{e}^{i s X_{s}} X \mathrm{e}^{-i s X_{s}}\right) \quad \text { and } \quad X \mapsto \omega\left(\mathrm{e}^{i s Z_{p}} X \mathrm{e}^{-i s Z_{p}}\right)
$$

are $\alpha_{t}$-KMS states at the same inverse temperature $\beta$. All such states are unitarily equivalent to the original one. By (10), $\omega$ is invariant under all these local unitary transformations

$$
\omega(X)=\omega\left(\mathrm{e}^{i s X_{s}} X \mathrm{e}^{-i s X_{s}}\right)=\omega\left(\mathrm{e}^{i s Z_{p}} X \mathrm{e}^{-i s Z_{p}}\right) .
$$

We now compute the $\alpha_{t}$-KMS states. It suffices to compute the value of $\omega$ on any monomial in the Pauli matrices, i.e. on any observable of the kind

$$
X=\prod_{j \in \Lambda} \sigma_{j}^{\varepsilon_{j}}, \quad \varepsilon_{j} \in\{x, y, z\}
$$


Using the invariances (44) with the value $s=\pi$, we find that

$$
\omega(X)=\omega\left(\prod_{j \in \Lambda} \sigma_{j}^{\varepsilon_{j}}\right)=0,
$$

unless $X$ is a product of star and plaquette observables. This implies that $\omega$ is completely determined by its values on the commutative algebra generated by the $\mathcal{A}_{\mathrm{x}}$ and $\mathcal{A}_{\mathrm{z}}$. The restriction of $\omega$ to this algebra is, however, a classical equilibrium state of a free Ising Hamiltonian at inverse temperature $\beta$. It is well-known that there is only one such state which has a product structure fully determined by the expectations

$$
\omega\left(X_{s}\right)=\omega\left(Z_{p}\right)=\tanh \left(\frac{\beta}{2}\right) .
$$

\section{The open Ising and Kitaev models}

We shall for both models consider noise originating from a coupling of the atomic spins to a surrounding heat bath. In order to keep things as simple as possible, we shall assume that each spin has its private heat bath which is moreover independent from these of the other spins. All baths are assumed to be identical. Moreover, we shall restrict ourselves to the Markovian approximation applying directly the weak coupling limit of Davies [6].

Before treating the Ising and Kitaev models, we briefly sketch the general setup and properties of Davies generators. A small system is coupled to a collection of heat baths leading to the global Hamiltonian

$$
H=H^{\text {sys }}+H^{\text {bath }}+H^{\text {int }} \quad \text { with } \quad H^{\text {int }}=\sum_{\alpha} S_{\alpha} \otimes f_{\alpha},
$$

where the $S_{\alpha}$ are system operators and the $f_{\alpha}$ bath operators. The main ingredients are the Fourier transforms $\hat{h}_{\alpha}$ of the autocorrelation functions of the $f_{\alpha}$. The function $\hat{h}_{\alpha}$ describes the rate at which the coupling is able to transfer an energy difference $\omega$ from the bath to the system. Often a minimal coupling to the bath is chosen, minimal in the sense that the interaction part of the Hamiltonian is as simple as possible but still addresses all energy levels of the system Hamiltonian in order to produce finally an ergodic reduced dynamics. The necessary and sufficient condition for ergodicity is [26, 27]

$$
\left\{S_{\alpha}, H^{\mathrm{sys}}\right\}^{\prime}=\mathbb{C} \mathbb{1},
$$


i.e. no system operator apart from the multiples of the identity commutes with all the $S_{\alpha}$ and $H^{\text {sys }}$.

We begin by introducing the Fourier decompositions of the $S_{\alpha}$ 's as they evolve in time under the system evolution

$$
\mathrm{e}^{i t H^{\text {sys }}} S_{\alpha} \mathrm{e}^{-i t H^{\text {sys }}}=\sum_{\omega} S_{\alpha}(\omega) \mathrm{e}^{-i \omega t}
$$

Here the $\omega$ are the Bohr frequencies of the system Hamiltonian. From selfadjointness we have the relation

$$
S_{\alpha}(-\omega)=S_{\alpha}(\omega)^{\dagger}
$$

The weak coupling limit procedure then returns the following equation for the evolution of the spin system in Heisenberg picture

$$
\begin{aligned}
\frac{d X}{d t} & =i\left[H^{\mathrm{sys}}, X\right]+\mathcal{L}_{\mathrm{dis}}(X)=: \mathcal{L}(X) \\
\mathcal{L}_{\mathrm{dis}}(X) & =\frac{1}{2} \sum_{\alpha} \sum_{\omega} \hat{h}_{\alpha}(\omega)\left(S_{\alpha}^{\dagger}(\omega)\left[X, S_{\alpha}(\omega)\right]+\left[S_{\alpha}^{\dagger}(\omega), X\right] S_{\alpha}(\omega)\right)
\end{aligned}
$$

For thermal baths one has moreover the relation

$$
\hat{h}_{\alpha}(-\omega)=\mathrm{e}^{-\beta \omega} \hat{h}_{\alpha}(\omega)
$$

which is a consequence of the KMS condition. The operator $\mathcal{L}$ generates a semigroup of completely positive identity preserving transformations of the spin system. It describes the reduced dynamics in the Markovian approximation and enjoys the following properties

- The canonical Gibbs state with density matrix

$$
\rho_{\beta}=\frac{\mathrm{e}^{-\beta H^{\mathrm{sys}}}}{\operatorname{Tr}\left(\mathrm{e}^{-\beta H^{\mathrm{sys}}}\right)}
$$

is a stationary state for the semigroup, i.e.

$$
\operatorname{Tr}\left(\rho_{\beta} \mathrm{e}^{t \mathcal{L}}(X)\right)=\operatorname{Tr}\left(\rho_{\beta} X\right)
$$


- The semigroup is relaxing, meaning that for any initial state $\rho$ of the system

$$
\lim _{t \rightarrow \infty} \operatorname{Tr}\left(\rho \mathrm{e}^{t \mathcal{L}}(X)\right)=\operatorname{Tr}\left(\rho_{\beta} X\right) .
$$

- Furthermore, the generator satisfies the detailed balance condition, often called reversibility. Writing $\delta(X):=\left[H^{\text {sys }}, X\right]$,

$$
\left[\delta, \mathcal{L}_{\text {dis }}\right]=0 \quad \text { and } \quad \operatorname{Tr}\left(\rho_{\beta} Y^{\dagger} \mathcal{L}_{\text {dis }}(X)\right)=\operatorname{Tr}\left(\rho_{\beta}\left(\mathcal{L}_{\text {dis }}(Y)\right)^{\dagger} X\right) .
$$

The last equation expresses the self-adjointness of the generator with respect to the scalar product defined by the equilibrium state. The space of observables equipped with the scalar product

$$
\langle X, Y\rangle_{\beta}:=\operatorname{Tr} \rho_{\beta} X^{\dagger} Y
$$

is called the Liouville space and the generator of the reduced dynamics is a normal matrix on that space, i.e. the Hermitian and skew-Hermitian parts of the generator commute.

\subsection{Dissipative generators for Ising and Kitaev models}

The eigenstates and eigenvalues of the Ising Hamiltonian are conveniently labelled by the excited bonds and one more dichotomic variable which takes the twofold degeneracy into account. This last could be the eigenvalues of $\mathbf{Z}=\sigma_{1}^{z}$. An excited bond is often called kink and can be seen as a quasiparticle. In the model on a ring, kinks always appear in pairs due to the topology of the system. The two dimensional Kitaev model will admit a very similar description in terms of excited plaquette and star observables now called anyons.

More precisely, for the Ising model on a ring, the eigenstates of the Hamiltonian are written as $\left|b_{1}, b_{2}, \ldots, b_{2 n} ; z\right\rangle$, they have energy

$$
H^{\text {Ising }}\left|b_{1}, b_{2}, \ldots, b_{2 n} ; z\right\rangle=(2 n-N)\left|b_{1}, b_{2}, \ldots, b_{2 n} ; z\right\rangle
$$

The interaction Hamiltonian is chosen as

$$
H^{\mathrm{int}}=\sum_{j=1}^{N} \sigma_{j}^{x} \otimes f_{j}
$$


where $f_{j}$ is a self-adjoint field of the $j$-th bath and all the baths are isomorphic.

The Bohr frequencies of the Ising Hamiltonian are $0, \pm 2, \pm 4, \ldots$. Only the Bohr frequencies 0 and \pm 2 contribute to the Davies generator (53) due to the choice of the interaction. We compute the Fourier components of the $\sigma_{j}^{x}$ 's

$$
\mathrm{e}^{i t H^{\mathrm{spin}}} \sigma_{j}^{x} \mathrm{e}^{-i t H^{\mathrm{spin}}}=\mathrm{e}^{-2 i t} a_{j}+\mathrm{e}^{2 i t} a_{j}^{\dagger}+a_{j}^{0} .
$$

The operators $a_{j}$ and $a_{j}^{0}$ are given in terms of projection operators

$$
P_{j}^{0}=\frac{1}{2}\left(\mathbb{1}-Z_{b} Z_{b^{\prime}}\right) \quad \text { and } \quad P_{j}^{ \pm}=\frac{1}{4}\left(\mathbb{1} \mp Z_{b}\right)\left(\mathbb{1} \mp Z_{b^{\prime}}\right)
$$

with $j=b \cap b^{\prime}$ :

$$
\begin{aligned}
& a_{j}^{0}=P_{j}^{0} \sigma_{j}^{x} P_{j}^{0}=P_{j}^{0} \sigma_{j}^{x}=\sigma_{j}^{x} P_{j}^{0} \\
& a_{j}=P_{j}^{-} \sigma_{j}^{x} P_{j}^{+}=P_{j}^{-} \sigma_{j}^{x}=\sigma_{j}^{x} P_{j}^{+} .
\end{aligned}
$$

The Davies operators allow for an interpretation in terms of kinks. Consider two bonds $b$ and $b^{\prime}$ having a site $j$ in common. The operator $a_{j}^{0}$ kills states for which both $b$ and $b^{\prime}$ are either empty or occupied and exchanges the occupations if only one of them is. The operators $a_{j}^{+}$and $a_{j}^{-}$create and annihilate states with two kinks meeting at $j$ respectively and kill the other states. This leads us to the master equation in Heisenberg picture

$$
\begin{aligned}
& \frac{d X}{d t}=i\left[H^{\text {Ising }}, X\right] \\
& +\frac{1}{2} \sum_{j=1}^{N}\left\{\hat{h}(2)\left(a_{j}^{\dagger}\left[X, a_{j}\right]+\left[a_{j}^{\dagger}, X\right] a_{j}+\mathrm{e}^{-2 \beta} a_{j}\left[X, a_{j}^{\dagger}\right]+\mathrm{e}^{-2 \beta}\left[a_{j}, X\right] a_{j}^{\dagger}\right)\right. \\
& \left.\quad-\hat{h}(0)\left[a_{j}^{0},\left[a_{j}^{0}, X\right]\right]\right\}
\end{aligned}
$$

For Kitaev's model on a torus, the eigenstates of the Hamiltonian are written as $\left|p_{1}, p_{2}, \ldots, p_{2 m} ; s_{1}, s_{2}, \ldots, s_{2 n} ; z_{1}, z_{2}\right\rangle$. The labels $z_{1}$ and $z_{2}$ refer to the eigenvalues of the $z$-components of the encoded qubits while the $p_{j}$ and $s_{k}$ point to the excited plaquette and star observables. Such an eigenstate has energy

$$
\begin{aligned}
H^{\mathrm{Kitaev}} & \left|p_{1}, p_{2}, \ldots, p_{2 m} ; s_{1}, s_{2}, \ldots, s_{2 n} ; z_{1}, z_{2}\right\rangle \\
\quad & =(2 m+2 n-N)\left|p_{1}, p_{2}, \ldots, p_{2 m} ; s_{1}, s_{2}, \ldots, s_{2 n} ; z_{1}, z_{2}\right\rangle
\end{aligned}
$$


The interaction Hamiltonian is chosen as

$$
H^{\mathrm{int}}=\sum_{j=1}^{N} \sigma_{j}^{x} \otimes f_{j}+\sum_{j=N+1}^{2 N} \sigma_{j}^{z} \otimes f_{j}
$$

where $f_{j}$ is a self-adjoint field of the $j$-th bath and all the baths are isomorphic.

The Bohr frequencies of the Kitaev Hamiltonian are $0, \pm 2, \pm 4, \ldots$. Again, only the Bohr frequencies 0 and \pm 2 contribute to the Davies generator (53) due to the choice of the interaction and we compute the Fourier decompositions of the spins as they evolve in time

$$
\begin{aligned}
& \mathrm{e}^{i t H^{\mathrm{spin}}} \sigma_{j}^{x} \mathrm{e}^{-i t H^{\mathrm{spin}}}=\mathrm{e}^{-2 i t} a_{j}+\mathrm{e}^{2 i t} a_{j}^{\dagger}+a_{j}^{0} \\
& \mathrm{e}^{i t H^{\mathrm{spin}}} \sigma_{j}^{z} \mathrm{e}^{-i t H^{\mathrm{spin}}}=\mathrm{e}^{-2 i t} b_{j}+\mathrm{e}^{2 i t} b_{j}^{\dagger}+b_{j}^{0} .
\end{aligned}
$$

The operators $a$ and $b$ are again given in terms of projection operators

$$
P_{j}^{0}=\frac{1}{2}\left(\mathbb{1}-Z_{p} Z_{p^{\prime}}\right) \quad \text { and } \quad P_{j}^{ \pm}=\frac{1}{4}\left(\mathbb{1} \mp Z_{p}\right)\left(\mathbb{1} \mp Z_{p^{\prime}}\right)
$$

with $j=p \cap p^{\prime}$. We need a second set of projections associated with the stars

$$
R_{j}^{0}=\frac{1}{2}\left(\mathbb{1}-X_{s} X_{s^{\prime}}\right) \quad \text { and } \quad R_{j}^{ \pm}=\frac{1}{4}\left(\mathbb{1} \mp X_{s}\right)\left(\mathbb{1} \mp X_{s^{\prime}}\right)
$$

with $j=s \cap s^{\prime}$. Then

$$
\begin{aligned}
& a_{j}^{0}=P_{j}^{0} \sigma_{j}^{x} P_{j}^{0}=P_{j}^{0} \sigma_{j}^{x}=\sigma_{j}^{x} P_{j}^{0} \\
& a_{j}=P_{j}^{-} \sigma_{j}^{x} P_{j}^{+}=P_{j}^{-} \sigma_{j}^{x}=\sigma_{j}^{x} P_{j}^{+} .
\end{aligned}
$$

and

$$
\begin{aligned}
& b_{j}^{0}=R_{j}^{0} \sigma_{j}^{z} R_{j}^{0}=R_{j}^{0} \sigma_{j}^{z}=\sigma_{j}^{z} R_{j}^{0} \\
& b_{j}=R_{j}^{-} \sigma_{j}^{z} R_{j}^{+}=R_{j}^{-} \sigma_{j}^{z}=\sigma_{j}^{z} R_{j}^{+} .
\end{aligned}
$$

The Davies operators allow for a similar interpretation as in the Ising case in terms of anyons. This leads us finally to the master equation in Heisenberg 
picture

$$
\begin{aligned}
& \frac{d X}{d t}=i\left[H^{\text {Kitaev }}, X\right] \\
& +\frac{1}{2} \sum_{j=1}^{N}\left\{\hat{h}(2)\left(a_{j}^{\dagger}\left[X, a_{j}\right]+\left[a_{j}^{\dagger}, X\right] a_{j}+\mathrm{e}^{-2 \beta} a_{j}\left[X, a_{j}^{\dagger}\right]+\mathrm{e}^{-2 \beta}\left[a_{j}, X\right] a_{j}^{\dagger}\right)\right. \\
& \left.\quad-\hat{h}(0)\left[a_{j}^{0},\left[a_{j}^{0}, X\right]\right]\right\} \\
& +\frac{1}{2} \sum_{j=1}^{N}\left\{\hat{h}(2)\left(b_{j}^{\dagger}\left[X, b_{j}\right]+\left[b_{j}^{\dagger}, X\right] b_{j}+\mathrm{e}^{-2 \beta} b_{j}\left[X, b_{j}^{\dagger}\right]+\mathrm{e}^{-2 \beta}\left[b_{j}, X\right] b_{j}^{\dagger}\right)\right. \\
& \left.\quad-\hat{h}(0)\left[b_{j}^{0},\left[b_{j}^{0}, X\right]\right]\right\} .
\end{aligned}
$$

Both the Davies generators for the Ising and Kitaev models extend to generators of completely positive identity preserving semigroups on the infinite chain and the infinite staggered lattice. This easily follows from the locality of the elements in the generator and the non-propagation of both the Hamiltonian and dissipative parts in the generators.

\subsection{Dynamics of the qubit observables}

In order to treat both the Ising and Kitaev models in a unified way it is useful to introduce the notation $\mathcal{A}_{\mathrm{ab}}$ for the Abelian algebras of observables. In the Ising case $\mathcal{A}_{\mathrm{ab}}=\mathcal{A}_{\mathrm{z}}$ and for the Kitaev model $\mathcal{A}_{\mathrm{ab}}=\mathcal{A}_{\mathrm{xz}}$. In both cases we denote by $\mathcal{C}$ the commutant of $\mathcal{A}_{\mathrm{ab}}$ in the respective spin algebras, so $\mathcal{C}=\mathcal{Q} \mathcal{A}_{\mathrm{z}}$ for Ising and $\mathcal{C}=\mathcal{Q}_{1} \mathcal{Q}_{2} \mathcal{A}_{\mathrm{x} z}$ for Kitaev. The algebras $\mathcal{Q}$ and $\mathcal{Q}_{1,2}$ are the qubit algebras introduced in (13) and (25). So, e.g. in the Ising case any element of $\mathcal{C}$ can be written as linear combination of $\mathbb{1}$ and the qubit observables $\mathrm{X}, \mathrm{Y}$ and $\mathrm{Z}$ with coefficients in $\mathcal{A}_{\mathrm{ab}}$. Let us denote generically by $\{Q\}$ the bases of the one or two-qubit spaces, so $\{Q\}=\{\mathbb{1}, X, Y, Z\}$ in the Ising case.

We consider first more general qubit observables $\{\tilde{Q}\}=\{\mathbb{1}, \tilde{X}, \tilde{Y}, \tilde{Z}\}$ which enjoy the following stability property: $[\tilde{Q}, A]=0$ for all $A \in \mathcal{A}_{\mathrm{ab}}$, i.e. $\{\tilde{\mathrm{Q}}\} \subset$ $\mathcal{C}$. The physical meaning of this condition is that the $\{\tilde{Q}\}$ are not only 
invariant under the Ising or Kitaev dynamics but also under a whole class of perturbed dynamics of the form

$$
H^{\prime \text { Ising }}:=-\sum_{b} J_{b} Z_{b} \quad \text { and } \quad H^{\prime \text { Kitaev }}:=-\sum_{s} J_{s}^{x} X_{s}-\sum_{p} J_{p}^{z} Z_{p} .
$$

In these formulas, the coupling constants are arbitrary but strictly larger than 0 . Remark that these perturbed models return precisely the same ground states as the original ones. In fact it will suffice to consider encodings of the type

$$
\tilde{\mathrm{Q}}=\mathrm{Q} F_{\mathrm{Q}}, \quad F_{\mathrm{Q}} \in \mathcal{A}_{\mathrm{ab}}
$$

where $\{Q\}$ is the choice mentioned above. In order to satisfy the qubit relations we have to impose on the functions $F$ that

$$
F_{\mathrm{X}} F_{\mathrm{Y}}=F_{\mathrm{Z}}, \quad F_{\mathrm{Q}}=F_{\mathrm{Q}}^{\dagger}, \quad F_{\mathrm{Q}}^{2}=\mathbb{1}, \quad \text { and } F_{\mathbb{1}}=\mathbb{1} .
$$

In order to quantify the quality of a memory we are looking for the shortest decay time of the time autocorrelation functions

$$
\langle\tilde{\mathbf{Q}}(t), \tilde{\mathbf{Q}}\rangle_{\beta}=\operatorname{Tr}\left(\rho_{\beta} \mathrm{e}^{t \mathcal{L}}(\tilde{\mathrm{Q}}) \tilde{\mathrm{Q}}\right)=\operatorname{Tr}\left(\rho_{\beta} \mathrm{e}^{t \mathcal{L}_{\mathrm{dis}}}(\tilde{\mathrm{Q}}) \tilde{\mathrm{Q}}\right)=\|\tilde{\mathbf{Q}}(t / 2)\|_{\beta}^{2}
$$

This chain of equalities follows from the properties of the generator of the reduced dynamics, derived at the beginning of Section 3. The time autocorrelation functions measure how well a system encoded with a message at time 0 has retained its information up to time $t$. The rightmost $\tilde{Q}$ in the product can be seen as a polarisation of the system corresponding to writing a message at $t=0$ while the leftmost is the readout at time $t$.

We shall now compute the action of the reduced dynamics on elements of the form $\tilde{Q}=\mathrm{Q} F_{\mathrm{Q}}$ with $F_{\mathrm{Q}}$ as above and show that

$$
\mathrm{e}^{t \mathcal{L}}(\tilde{\mathrm{Q}})=\mathrm{Q} \mathrm{e}^{t \tilde{\mathcal{L}}_{\mathrm{Q}}}\left(F_{\mathrm{Q}}\right)=\mathrm{e}^{t \tilde{\mathcal{L}}_{\mathrm{Q}}}\left(F_{\mathrm{Q}}\right) \mathrm{Q}
$$

with

$$
\mathrm{e}^{t \tilde{\mathcal{L}}_{\mathrm{Q}}}: \mathcal{A}_{\mathrm{ab}} \rightarrow \mathcal{A}_{\mathrm{ab}}
$$

a contracting semigroup, hence

$$
\langle\tilde{Q}(t), \tilde{Q}\rangle_{\beta}=\left\langle\mathrm{e}^{t \tilde{\mathcal{L}}_{\mathrm{Q}}}\left(F_{\mathrm{Q}}\right), F_{\mathrm{Q}}\right\rangle_{\beta}
$$


In order to verify (82) we need some relations

$$
\begin{aligned}
& \sigma_{j}^{\alpha} F \sigma_{j}^{\alpha} \in \mathcal{A}_{\mathrm{ab}} \text { whenever } F \in \mathcal{A}_{\mathrm{ab}} \\
& \sigma_{j}^{\alpha} \mathrm{Q} \sigma_{j}^{\alpha}=g_{\mathrm{Q}}^{\alpha}(j) \mathrm{Q} \text { with } g_{\mathrm{Q}}^{\alpha}(j) \in\{ \pm 1\} .
\end{aligned}
$$

Consider a typical contribution to the generator

$$
\mathcal{K}(X):=a_{j} X a_{j}^{\dagger}-\frac{1}{2} a_{j} a_{j}^{\dagger} X-\frac{1}{2} X a_{j} a_{j}^{\dagger}
$$

with

$$
a_{j}=P_{j}^{-} \sigma_{j}^{x} P_{j}^{+}, \quad P_{j}^{ \pm} \in \mathcal{A}_{\mathrm{ab}} .
$$

For $F \in \mathcal{A}_{\mathrm{ab}}$ we obtain

$$
\begin{aligned}
\mathcal{K}(\mathrm{Q} F) & =P_{j}^{-} \sigma_{j}^{x} \mathrm{Q} \sigma_{j}^{x} \sigma_{j}^{x} F \sigma_{j}^{x} P_{j}^{-}-\mathrm{Q} P_{j}^{-} F \\
& =\mathrm{Q}\left(P_{j}\left(g_{\mathrm{Q}}^{x}(j) \sigma_{j}^{x} F \sigma_{j}^{x}-F\right)\right)=: \mathrm{Q} \tilde{\mathcal{K}}_{\mathrm{Q}}(F) .
\end{aligned}
$$

Clearly $\tilde{\mathcal{K}}_{\mathrm{Q}}(F) \in \mathcal{A}_{\mathrm{ab}}$. It now suffices to add all contributions in order to show (82). In this way we obtain the following expressions for the reduced generators $\tilde{\mathcal{L}}_{\mathrm{Q}}$

$$
\begin{aligned}
& \tilde{\mathcal{L}}_{\mathrm{Q}}^{\text {Ising }}(F)= \sum_{j=1}^{N} D_{j}^{x}\left(g_{\mathrm{Q}}^{x}(j) \sigma_{j}^{x} F \sigma_{j}^{x}-F\right) \\
& \tilde{\mathcal{L}}_{\mathrm{Q}}^{\text {Kitaev }}(F)= \sum_{j=1}^{N}\left\{D_{j}^{x}\left(g_{\mathrm{Q}}^{x}(j) \sigma_{j}^{x} F \sigma_{j}^{x}-F\right)\right. \\
&\left.+D_{j}^{z}\left(g_{\mathrm{Q}}^{z}(j) \sigma_{j}^{z} F \sigma_{j}^{z}-F\right)\right\} \\
& D_{j}^{x}=\hat{h}(-2) P_{j}^{-}+\hat{h}(2) P_{j}^{+}+\hat{h}(0) P_{j}^{0} \\
& D_{j}^{z}=\hat{h}(-2) R_{j}^{-}+\hat{h}(2) R_{j}^{+}+\hat{h}(0) R_{j}^{0} .
\end{aligned}
$$

Finally, let us analyse the evolution of some special observables in Kitaev's model. Consider observables $\tilde{X}_{1}$ and $\tilde{Z}_{1}$ of the first qubit given by

$$
\tilde{\mathrm{X}}_{1}=\mathrm{X}_{1} F^{x}, \quad \tilde{\mathrm{Z}}_{1}=\mathrm{Z}_{1} F^{z}
$$


with $F^{x} \in \mathcal{A}_{x}, F^{z} \in \mathcal{A}_{z}$, and $\mathrm{X}_{1}, \mathrm{Z}_{1}$ as in (25). It suffices to consider one of them, e.g. $\tilde{Z}_{1}$. It turns out that the following Hamiltonian and coupling with the environment generate the same time evolution of this observable

$$
H_{z}^{\mathrm{Kitaev}}=-\sum_{\mathrm{p}} Z_{\mathrm{p}} \quad \text { and } \quad H^{\mathrm{int}}=\sum_{j=1}^{N} \sigma_{j}^{x} \otimes f_{j} .
$$

More precisely, we have

$$
\mathrm{e}^{\mathcal{L}^{\mathrm{Kitaev}} t} \tilde{Z}_{1}=\mathrm{e}^{\mathcal{L}^{\mathrm{Kitaev} z} t} \tilde{\mathbf{Z}}_{1}
$$

where $\mathcal{L}^{\mathrm{Kitaev}_{z}}$ is solely determined by Davies operators of $a$-type

$$
a_{j}^{0}=\sigma_{j}^{x} P_{j}^{0} \quad \text { and } \quad a_{j}=\sigma_{j}^{x} P_{j}^{+} .
$$

Similar relations hold for the second observable. This can be interpreted as follows: with this choice of basic observables, bit and phase evolve separately according to the same evolution up to an exchange of $\sigma_{x}$ and $\sigma_{z}$. This property is then inherited by the reduced generators. Such a separation is not surprising. It simply means that such a choice of qubit observables is compatible with the structure of the quantum code behind Kitaev's model. Namely, the subspace of ground states constitutes a so-called CSS code [28, 29]. The characteristic feature of this class of codes is that the recovery procedure, aiming at restoring the initial state of the qubit after the attack by the noise, is divided into two stages: first correcting bit errors and then phase errors. The correction procedures are again the same, modulo $\sigma_{z} \leftrightarrow \sigma_{x}$ exchange.

The aim of this paper was to analyse the static and dynamical structure of Kitaev's model in 2D, using the analogy with the Ising ring or chain. More involved models in 3 and $4 \mathrm{D}$ should be addressable in a similar way. A further topic is determining the spectral properties of the reduced semigroup generators, especially the behaviour of relaxation times as a function of the system size. Such questions are important in order to decide whether such systems are reasonable candidates for stable quantum memories. This will be dealt with in a forthcoming paper.

Acknowledgements: We would like to thank J. Preskill, P. Horodecki and R. Horodecki for stimulating discussions. This work is supported by the Polish-Flemish bilateral grant BIL 05/11 (MF), the Polish Ministry of Science 
and Information Technology - grant PBZ-MIN-008/P03/2003 (RA and MH), EU Integrated Project Qubit Applications QAP -IST directorate contract 015848 (RA) and EC IP SCALA (MH).

\section{References}

[1] Shor P W 1996 Fault-tolerant quantum computation in Proceedings of the 37th Symposium on Foundations of Computing, Los Alamitos, CA (IEEE Computer Society Press) pp 56-65 quant-ph/9605011

[2] Knill E, Laflamme R, and Żurek W H 1998 Resilient quantum computation Science 279 342-345

[3] Aharonov D and Ben-Or M 1997 Fault-tolerant quantum computation with constant error rate in Proc. of the 29th Annual ACM Symposium on Theory of Computing (STOC) 1997 176-188 quant-ph/9910081

[4] Gottesman D 2000 Fault-tolerant quantum computation with local gates J. Mod. Opt. 47 333-345 quant-ph/9903099

[5] Preskill J 1998 Reliable quantum computers Proc. Roy. Soc. Lond. A 454 385-410 quant-ph/9705031

[6] Davies E B 1974 Markovian master equations Commun. Math. Phys. 39 91-110

[7] Alicki R and Lendi K 1993 Quantum Dynamical Semigroups and Applications (Berlin: Springer)

[8] Breuer H P and Petruccione F 2002 The Theory of Open Quantum Systems (Oxford: Oxford University Press)

[9] Alicki R, Horodecki M, Horodecki P, and Horodecki R 2001 Dynamical description of quantum computing: Generic nonlocality of quantum noise Phys. Rev. A 65062101 quant-ph/0105115

[10] Alicki R, Lidar D, and Zanardi P 2006 Internal consistency of faulttolerant quantum error correction in light of rigorous derivations of the quantum markovian limit Phys. Rev. A 73052311 quant-ph/0506201 
[11] Terhal B M and Burkard G 2005 Fault-tolerant quantum computation for local non-markovian noise Phys. Rev. A 71012336

quant-ph/0402104

[12] Alicki R 2004 Comments on "Fault-tolerant quantum computation for local non-markovian noise" quant-ph/0402139

[13] Aharonov D, Kitaev A, and Preskill J 2006 Fault-tolerant quantum computation with long-range correlated noise Phys. Rev. Lett. 96050504 quant-ph/0510231

[14] Kitaev A Y 2003 Fault-tolerant quantum computation by anyons Annals Phys. 303 2-30 quant-ph/9707021

[15] Dennis E, Kitaev A, Landahl A, and Preskill J 2002 Topological quantum memory J. Math. Phys. 43 4452-4505 quant-ph/0110143

[16] Robinson D W and Bratteli 1997 Operator Algebras and Quantum Statistical Mechanics 2 (Berlin: Springer Verlag)

[17] Pusz W and Woronowicz S L 1978 Passive states and KMS states for general quantum systems Commun. Math. Phys. 58 273-290

[18] Martin P A 1977 On the stochastic dynamics of Ising models J. Stat. Phys. 16 149-168

[19] Buffet E, De Smedt P, and Pulé J V 1984 The dynamics of the open bose gas Ann. Phys. 155 269-304

[20] Pirogov S A and Sinai Ya G 1975 and 1976 Phase diagrams of classical lattice systems Theor. Math. Phys. 25 1185-1192 and 26 39-49

[21] Joos E, Zeh H D, Kiefer C, Giulini D, Kupsch J, and Stamatescu I O 2003 Decoherence and the Appearance of a Classical World in Quantum Theory, Second Edition (Berlin: Springer)

[22] Spohn H 1989 Ground state(s) of the spin-boson hamiltonian Commun. Math. Phys. 123 277-304

[23] Lloyd S, Rahn B, and Ahn C 1999 Robust quantum computations by simulations quant-ph/9912040 
[24] Lloyd S and Zanardi P 2003 Topological protection and quantum noiseless subsystems Phys. Rev. Lett. 90067902 quant-ph/0208132

[25] Alicki R and Horodecki M 2006 Can one build a quantum hard drive? A no-go theorem for storing quantum information in equilibrium systems quant-ph/0603260

[26] Spohn H 1977 An algebraic condition for the approach to equilibrium of an open $N$-level system Lett. Math. Phys. 2 33-38

[27] Frigerio A 1977 Stationary states of quantum dynamical semigroups Commun. Math. Phys. 63 269-276

[28] Steane A 1996 Multiple particle interference and quantum error correction Proc. Roy. Soc. Lond. A 452 2551-2577

[29] Calderbank A R and Shor P W 1996 Good quantum error-correcting codes exist Phys. Rev. A 54 1098-1105 